\title{
Lumen
}

Selected Proceedings from the Canadian Society for Eighteenth-Century Studies

\section{Le personnage de Philoctète dans l'Oedipe de Voltaire : un signe avant-coureur}

\section{Georges-L. Bérubé}

\section{Volume 13, 1994}

URI : https://id.erudit.org/iderudit/1012521ar

DOI : https://doi.org/10.7202/1012521ar

Aller au sommaire du numéro

Éditeur(s)

Canadian Society for Eighteenth-Century Studies / Société canadienne d'étude du dix-huitième siècle

ISSN

1209-3696 (imprimé)

1927-8284 (numérique)

Découvrir la revue

Citer cet article

Bérubé, G.-L. (1994). Le personnage de Philoctète dans l'Oedipe de Voltaire : un signe avant-coureur. Lumen, 13, 61-69. https://doi.org/10.7202/1012521ar 


\section{Le personnage de Philoctète dans 1'CEdipe de Voltaire: un signe avant-coureur}

Le personnage de Philoctète dans la première tragédie d'Arouet de Voltaire, CEdipe représentée en 1718 , a toujours fait problème, du fait qu'il constitue une sorte de faille dans l'ensemble cohérent que devait être toute tragédie classique. En effet, pourquoi le dramaturge fait-il apparaître le personnage pendant les trois premiers actes pour ensuite le faire disparaitre complètement sans explication adéquate? En outre, comment expliquer qu'un public, aussi averti que celui qui fréquentait alors le Théâtre Français, ait applaudi une œuvre avec un vice aussi évident? D'ailleurs, Philoctète, taxé d"épisodique' par Desnoirresterre et la critique traditionnelle, continue à gêner la critique contemporaine, comme l'indique le fait que René Pomeau, dans La Religion de Voltaire, réussit à analyser la pièce sans mentionner le personnage qui occupe le centre de la scène pendant trois des cinq actes. Finalement, il est extraordinaire, pour ne pas dire tout à fait incompréhensible, de voir le jeune auteur dans sa cinquième Lettre sur CEdipe, publiée à la tête de l'édition de 1719, reconnaître le problème sans pour autant faire le moindre effort pour l'éliminer. Rappelons le passage en question:

Voici un défaut plus considérable qui n'est pas du sujet, et dont je suis seul responsable; c'est le personnage de Philoctète. Il semble qu'il ne soit venu à Thèbes que pour y être accusé; encore est-il soupçonné peut-être un peu légèrement. Il arrive au premier acte, et s'en retourne au troisième; on ne parle de lui que dans les trois premiers actes, et l'on n'en dit pas un seul mot dans les deux derniers. Il contribue un peu au nœud de la pièce, et le dénoûment [sic] se fait absolument sans lui. Ainsi, il paraît que ce sont deux tragédies dont l'une roule sur Philoctète et l'autre sur CEdipe.'

Voltaire, d'habitude si soucieux du respect des règles et surtout d'une règle aussi fondamentale que l'unité d'action, la viole ici sans vergogne, se contentant d'invoquer le peu de matière qu'offrait le sujet grec, de sorte que la présence de Philoctète à Thèbes et 'le souvenir d'amour' qu'évoquent Jocaste et Philoctète deviennent, selon l'expression même 
de l'auteur, un 'défaut nécessaire' (M. ii, 38). Dans son analyse de la pièce, L'CEdipe de Voltaire: introduction à une psycholecture, José-Michel Moureaux relève l'importance que doit avoir une telle faille dans la perspective qui est la sienne: '... il y a un véritable problème $d^{\prime}$ CEdipe - nous dirions presque un mystère puisque le premier à n'avoir pu le résoudre fut l'auteur lui-même - celui de sa "malfaçon."'2

Il ne s'agit évidemment pas pour nous de reprendre ici la brillante analyse de Moureaux, mais plutôt d'examiner dans quelle mesure ce personnage prétendument inutile peut être considéré l'annonce de quelque chose de nouveau.

Pour ceux qui n'auraient pas à la mémoire les trois actes auxquels nous faisons allusion, nous en retraçons ici les grandes lignes, surtout en ce qui concerne le personnage qui nous intéresse. De retour à Thèbes après une absence prolongée, Philoctète se voit obligé de se justifier devant son vieil ami Dimas, qui lui demande ce qui a bien pu le ramener dans ce lieu pestiféré. Dans l'édition de 1719, la question est posée à brûle pourpoint: 'Vous dans Thèbes, seigneur! Eh!, qu'y venez-vous faire?' (M. ii, 112). Un critique dans l'édition de Kehl fait la remarque suivante sur ce vers qui se trouve alors relégué parmi les variantes: 'Ce dernier hémistiche avertissait trop clairement de l'inutilité du rôle de Philoctète' (M. ii, 112). Si Philoctète, dans l'édition originale, répondait que c'était son amour pour Jocaste qui le rappelait à Thèbes, dans les éditions subséquentes, il esquive la question amoureuse pour ne parler que de la mort d'Hercule et des tombeaux qu'il souhaite lui élever. L'annonce de la mort de Laïus rallume son espoir de retrouver Jocaste, mais presque aussitôt il apprend que la reine a dû se remarier avec le vainqueur du sphinx. Ainsi, à la fin de la première scène, Philoctète a été un bon élément dans l'exposition de l'intrigue, mais, ce qui importe davantage, il a révélé son incapacité de jouer un rôle efficace dans la tragédie de Thèbes. De là, son intention de prier pour le salut du peuple.

Par deux fois dans la cinquième Lettre sur CEdipe citée plus tôt, Voltaire répète qu'il a 'voulu donner à Philoctète le caractère d'un héros.' Pourtant, dans la tragédie, force nous est de constater que tout ce que nous avons, c'est un discours sur des exploits passés:

A ses divins travaux [ceux d'Hercule] j'osai m'associer;

Je marchai près de lui, ceint du même laurier. (M. ii, 64)

A part sa fierté hautaine, aucun geste héroïque ne marque le comportement du personnage. Nous serions tenté de conclure qu'il vit de ses lauriers et qu'il est revenu à Thèbes pour prouver à quel point il méritait la main de la reine Jocaste. 
Lorsque s'ouvre le deuxième acte, un porte-parole du peuple accuse notre héros du meurtre du roi Laïus, car sa haine pour le feu roi était connue. Jocaste, quant à elle, se dit convaincue qu'un héros de cette trempe ne saurait être un vulgaire assassin. Ainsi s'engage un débat sur l'authenticité de la vertu de Philoctète, ce qui a pour but d'établir, hors de tout doute, la véracité de ses paroles: est-il, oui ou non, le héros qu'il dit être? Devant CEdipe, Philoctète consent à peine à se justifier, il exige qu'on le croie sur parole. Même scénario à l'acte III, lorsqu'il refuse d'obéir à Jocaste qui lui demande de fuir pour sauver sa vie. En fait, il propose même une hypothèse qui expliquerait sa présence dans la ville maudite:

Qui sait si sa clémence [celle du ciel], au sein de vos États,

Pour m'immoler à vous n'a point conduit mes pas? (M. ii, 84)

Mais nous savons qu'il n'en est rien puisque le Grand Prêtre est sur le point de désigner CEdipe comme le coupable. Survient alors une situation des plus embarrassantes: comment faire pour éliminer un personnage innocent devenu terriblement gênant? Dans l'édition de 1719, Philoctète décide tout simplement de quitter la ville condamnée pour chercher ailleurs des malheureux qu'il pourrait défendre, poursuivant par là le travail d'Hercule. Cette version a été modifiée à partir de l'édition de 1751 parce que, selon La Harpe dans son Commentaire littéraire, 'la sortie de Philoctète servait encore à isoler davantage son rôle, et à le détacher du reste de la pièce. ${ }^{3}$ La nouvelle version, en revanche, ne résoud pas tous les problèmes, loin de là. Philoctète commence par s'élever contre l'accusation du Grand Prêtre en la qualifiant d'injuste: 'Contre vos ennemis je vous offre mon bras.' Or, à la scène suivante lorsqu'il se retrouve seul en compagnie de Jocaste et CFdipe - sans que l'on sache très bien pourquoi - il fait volte-face et retire son appui à CEdipe: 'Si vous n'aviez, seigneur, à craindre que des rois,/Philoctète avec vous combattrait sous vos lois.' Conclusion: Philoctète choisit de ne pas se mesurer au pouvoir religieux, fût-il injuste et sanguinaire. Et le personnage disparaît sans plus de cérémonie. Il est vrai qu'à l'acte cinq EEdipe mentionne le nom de son rival lorsqu'il s'agit de choisir un successeur au trône, mais Philoctète est depuis longtemps oublié des spectateurs.

Bien que cette tragédie ait été assez bizarrement construite, la critique a tôt fait d'accepter l'auto-critique et les mauvaises raisons de son auteur, dû en grande partie au succès phénoménal que la pièce a connu, et sans doute aussi, comme l'écrit René Pomeau, parce qu'on y sentait quelque chose de neuf, que 'le vrai Voltaire est déjà dans CEdipe. ${ }^{4}$ Mais il a fallu attendre le travail de José-Michel Moureaux pour expliquer l'anomalie apparente de la pièce, pour comprendre que 'Philoctète, loin d'être un 
personnage épisodique (DES I, 141) se révélera un personnage essentiel, au moins aussi important que celui d'CEdipe. ${ }^{5}$ Toujours selon Moureaux, cette tragédie possède une 'unité très réelle ${ }^{6}$ qu' $^{\prime}$ il est possible de résume $r$ ainsi: ' $c$ 'est au tribunal du père que les deux fils comparaissent tour à tour, le premier pour un acquittement, le second pour sa condamnation. ${ }^{\prime 7}$ Cette interprétation - dite du 'niveau abyssal' - a le grand mérite d'expliciter enfin une réalité à laquelle Voltaire tenait mais qu'il ne pouvait s'expliquer, lui qui écrit qu'il y a dans CEdipe deux tragédies 'dont l'une roule sur Philoctète et l'autre sur CEdipe.'

Rassuré sur l'utilité profonde de Philoctète, nous pouvons maintenant aborder d'autres niveaux de signification de l'œuvre pour voir pourquoi ce personnage est si malaisé à classer, pourquoi il semble échapper à la fable à laquelle l'auteur veut l'intégrer. Acceptons donc, avec JoséMichel Moureaux, que Philoctète est essentiel, qu'il est celui 'où le créateur a très probablement mis le plus de lui-même ${ }^{\prime 8}$ et regardons-le maintenant de plus près. Un bon point de départ serait de prendre Voltaire au mot, lui qui faisait allusion à la 'tragédie' de Philoctète, et d'essayer de préciser en quoi consiste cette tragédie.

De prime abord, le personnage se présente comme plus grand que nature - idéal en quelque sorte - ayant pu s'associer aux travaux d'un demi-dieu, non, nous le précise Voltaire, en 'pauvre écuyer d'Hercule, qui n'avait d'autre mérite que d'avoir porté ses flèches,' mais en 'prince de la Grèce, fameux par ses exploits, compagnon d'Hercule, et de qui même les dieux avaient fait dépendre le destin de Troie' (M. ii, 37). Ne souffrant d'aucune tare morale, incapable d'envisager la moindre bassesse - ne dit-il pas 'Je ne sais point encor comme on manque de foi'? (M. ii, 84), Philoctète en est même arrivé à vaincre le plus grand obstacle à la gloire d'un héros, l'amour. C'est ce personnage, héros sans tache et chevalier fidèle, que le peuple ignorant va accuser de régicide. Pour vaine qu'elle soit, l'accusation n'est pas sans importance, puisqu'elle entraîne un jugement qui va faire resplendir l'innocence de la victime. Philoctète traverse donc l'épreuve n'ayant rien à se reprocher et il repart à la fin du troisième acte tout à fait indemne. Il ne nous semble pas que ce soit là une grande tragédie.

Elle ne peut sûrement pas se réduire à ce que Voltaire a qualifié du 'souvenir d'amour' qui existait entre le héros et Jocaste. Si Philoctète entretient pendant un bref instant au début de la pièce un regain d'espoir de posséder celle qu'il aimait, l'annonce du mariage entre CEdipe et Jocaste le rappelle à l'ordre et le confirme dans sa résolution de laisser de côté les affaires de cœur pour se consacrer entièrement à ses travaux de héros. Il ressort de tout ceci que la tragédie de Philoctète est aussi difficile à cerner que son rôle dans la pièce. La Harpe, dans son Commentaire littéraire, faisait le même genre de constatation. A la fin de l'acte II, 
après le vers 'Mais que Phorbas est lent pour mon impatience!' le critique prétend que le spectateur aussi devient impatient et qu'il n'y a pas de raison pour que la pièce piétine ainsi: 'Ce vers est une critique de la pièce, parce qu'en effet il n'y a guère de raison pour que Phorbas ne soit pas encore arrivé; mais il fallait faire cinq actes. ${ }^{\prime 9}$ Qu'est-ce à dire, sinon que la tragédie de Philoctète n'aboutit à rien et que celle d'Édipe, la vraie, en est alors retardée? La Harpe se fait plus cinglant encore dans son commentaire après le dernier vers de l'acte II: 'L'action n'a pas fait un pas dans cet acte, mais d'aussi beaux vers d'un jeune homme durent soutenir l'attention des spectateurs. ${ }^{10}$ Rien n'avance, tout piétine, pour la bonne et simple raison que Philoctète vit une tragédie que nous croyons être toute autre que celle d'CEdipe.

En effet, contrairement à la tragédie grecque qui est racontée dans la seconde moitié de la pièce, celle de Philoctète brille par son absence, c'est-à-dire que le héros souffre d'un manque, impossible à raconter, et son drame, c'est précisément ce qu'on lui a si souvent reproché: son inutilité. Par conséquent, le personnage étale ses souvenirs, ses errances, ses pertes (Hercule décédé, Jocaste mariée puis remariée, etc.) pour en arriver à souhaiter que les dieux prennent sa vie si elle peut sauver celle de la reine. Philoctète devient alors le signe de la non-appartenance. D'abord, il a été contraint de se déraciner et de partir en exil pour vaincre sa passion amoureuse - passion devenue criminelle puisque Jocaste appartenait désormais à Laïus - puis il a voyagé à travers le monde en compagnie d'Hercule, ce qui l'a empêché d'affronter le sphinx pour mériter la main de la reine:

Ah! pourquoi la fortune, à me nuire constante,

Emportait-elle ailleurs ma valeur imprudente?

Si le vainqueur du sphinx devait vous conquérir,

Fallait-il loin de vous ne chercher qu'à périr? (M. ii, 75)

L'idée récurrente dans le discours du héros c'est celle du destin manqué. Enfin, lorsque le drame de Thèbes roule davantage sur CEdipe, Philoctète reprend le chemin de l'exil, ce que l'édition originale de 1719 rendait on ne peut plus clair:

J'abandonne à jamais ces lieux remplis d'effroi;

Les chemins de la gloire y sont fermés pour moi.

Sur les pas du héros dont je garde la cendre,

Cherchons des malheureux que je puisse défendre. (M. ii, 116) 
Voilà la confirmation de son sort: il doit continuer d'errer de par le monde, mais voilà aussi la confirmation de sa raison d'être, de son utilité: il doit chercher à défendre les malheureux. Cette sortie de Philoctète n'a-t-elle pas quelque chose de prophétique? En effet, elle semble annoncer la carrière que Voltaire, le déraciné, choisira pour lui-même.

L'affirmation de Moureaux, à savoir que Philoctète est 'une création authentique' et 'probablement fantasme principal' de la pièce, prend ici tout son sens. Sans revenir sur le contenu du fantasme, nous croyons déceler dans cette 'création authentique' une projection du jeune Arouet. Or, qui est le dramaturge qui a osé mettre en scène un tel sujet? Les biographes nous aident à nous en faire une idée. René Pomeau, par exemple, écrivait récemment qu'à cette époque Arouet est 'un homme instable, improvisateur et versatile, qui très tôt a composé son personnage et l'a fait pulluler. ${ }^{11}$ Jusqu'au moment de l'écriture d'CEdipe, Arouet s'est contenté de jouer le rôle de l'aimable amuseur' en se promenant d'un salon parisien à un autre, d'une maison de campagne à une autre. Toujours selon René Pomeau: 'Il tourne prestement de petits vers malicieux. Il a de l'esprit, on le sait; une plaisanterie un peu trop maligne court-elle sans nom d'auteur, c'est à lui qu'on l'attribue. ${ }^{\prime 2}$ Notons toutefois que les petits vers sont souvent marqués d'une violence extrême. L'exemple le plus cité, le Regnante puero, se termine par ce vers: 'gallia mox peritura,' la Gaule va bientôt périr, mais il y en a bien d'autres, comme ceux-ci contre le Régent, rédigés en latin mais restés inédits:

Ce que tu es, je l'ai révélé à Apollon;

Bientôt, Apollon le révélera à tout le genre humain...

Melon et Rey [?] son collègue servent

Tes scandaleuses amours, en dignes ministres

D'un tel héros. (traduit par R. Pomeau) ${ }^{13}$

Quelle audace chez un jeune homme de juger le Régent et le gouvernement avec une telle virulence! Entend-il se poser en autorité morale qui ne peut tolérer la corruption qu'il voit partout autour? Mais de quel droit? N'est-ce pas plutôt sa prétention de contester l'autorité, toute autorité quelle qu'elle soit? Ou encore, n'est-ce pas une façon pour un déraciné - il se disait le bâtard de Rochebrune ne l'oublions pas - de justifier son existence, de réclamer sa place dans la société. C'est enfin le moment qu'il choisit pour baptiser son nouveau personnage du nom de Voltaire. Toutes ces questions, propres à stimuler l'intérêt du biographe, fournissent en plus des indices précieux sur ce qui préoccupe l'auteur. Elles indiquent que le discours prédominant dans les premiers écrits est marqué par une soif d'authenticité et de vérité: qu'il dénonce les mœurs 
scandaleuses de la cour, les abus de la religion, la catastrophe économique provoquée par le 'système' de Law, Voltaire cherche à faire valoir une morale épurée. Ajoutons à cela le fait qu'il est en perpétuel mouvement sans port d'attache. Pomeau écrit: 'Il n'a jamais eu jusqu'ici [en 1731] de domicile durable. Il vit dans des auberges, chez des amis, ou en pension chez des particuliers, mais toujours pour des périodes brèves: quelques jours, quelques semaines, tout au plus quelques mois. ${ }^{, 14}$ A la lumière de ces éléments biographiques, son Philoctète apparaît presque comme un alter ego. Mais gardons-nous d'identifier trop étroitement l'auteur et sa création. Tout au plus pouvons-nous dire que la biographie fournit des indications sur l'esprit qui a présidé à la création du nouveau personnage problématique qu'est Philoctète.

Revenons maintenant à la tragédie qui nous occupe. Le rôle de Philoctète pourrait se résumer de la façon suivante: celui du prophète malheureux qui doit témoigner de la vérité envers et contre tous. Étant donné la caution morale qui l'entoure depuis son entrée en scène - c'est en fait celui qui a pour mission d'élever des autels à Hercule - Philoctète s'impose aux personnages et aux spectateurs d'abord et avant tout par sa vertu. Malheureusement, la reconnaissance de cette vertu lors de la scène du jugement à l'acte III n'élimine pas la corruption dans Thèbes.

Somme toute, la tragédie de Philoctète, c'est d'être un prophète dans son propre pays, de témoigner d'une vertu qui, hélas, se révèle inutile. Ainsi s'explique, du moins le croyons-nous, le fait que l'action n'avance pas; en fait, il n'y a pas d'action, seuls sont rappelés des souvenirs, un passé rempli d'occasions perdues, seuls sont développés la description et le jugement d'un personnage authentique à la morale pure. S'il est inutile au niveau de l'action, la présence de Philoctète dans la cité damnée signifie par contre les valeurs dont elle est privée: l'authenticité, l'intégrité, la vérité. Sa présence est signe du refus du mal, c'est un jugement sévère à l'endroit d'une société en décomposition.

On pourrait nous objecter que le Grand Prêtre joue tous ces rôles dans la pièce. La question est de savoir s'il le fait avec efficacité. Or, ce que nous constatons, $c^{\prime}$ est que presque tous les personnages se lancent à tour de rôle dans une dénonciation du Grand Prêtre et de la religion. Qui ne se souvient de ces vers chocs?

Nos prêtres ne sont pas ce qu'un vain peuple pense,

Notre crédulité fait toute leur science. (M. ii, 93)

Bien entendu, le dénouement est dicté par la fable grecque de sorte que le Grand Prêtre a raison à la fin; la perception qu'en ont les spectateurs par contre ne peut être favorable. Celui qui aurait dû témoigner de la vérité est trop impliqué dans les institutions du pouvoir pour ne pas 
souffrir de leur contagion. Seul l'étranger Philoctète jouit d'une crédibilité absolue, lui qui affirme:

Le trône est un objet qui n'a pu me tenter:

Hercule à ce haut rang dédaignait de monter.

Toujours libre avec lui, sans sujets et sans maître,

J'ai fait des souverains, et n'ai point voulu l'être. (M. ii, 78)

Philoctète maintient son noble désintéressement jusqu'à la fin, ce qui ne sera pas le cas de Voltaire quelques années plus tard lorsqu'il écrit un petit divertissement appelé la Fête de Bélébat.

Là-dedans, il fait mourir le pauvre curé dans le but de prendre sa place. C'est alors que le bedeau chante ce vers absolument cocasse:

L'auteur d'CEdipe est devenu curé. (M. ii, 290)

Concluons en disant que Philoctète représente bien le signe avantcoureur d'un nouveau type de personnage, lequel sera éminemment voltairien. En effet, ce personnage intègre, qui incarne une morale épurée, désintéressée, qui est hors d'atteinte de la corruption politique, va se retrouver un peu partout dans l'œuvre de Voltaire. Au théâtre, pour ne citer que quelques exemples, nous trouvons Alvarez dans Alzire, Coucy dans Adélaïde du Guesclin et Cicéron dans Rome sauvée. Le personnage fait aussi son apparition dans d'autres genres, notamment dans $\mathrm{La}$ Henriade où il s'appelle Sully, avant de devenir plus tard Mornay; on le voit se manifester sous les traits du vieux quaker au début des Lettres philosophiques et du vieux sage d'Eldorado dans Candide. En général, ce type de personnage demeure épisodique, dans le sens qu'il se trouve en marge de l'action, à cause du rôle de conseiller que sa grande sagesse l'appelle à jouer. Dans la perspective didactique qui va en s'affirmant à mesure que Voltaire s'engage dans le combat philosophique, ce type de personnage permet à l'auteur de passer du niveau de la fable au niveau discursif, c'est-à-dire de porter sur l'œuvre qui s'écrit un jugement percutant.

GEORGES-L. BÉRUBÉ

Université York 


\section{Notes}

1 Voltaire, Lettres sur CEdipe, dans CEuvres complètes, édition Louis Moland (Paris: Garnier Frères, 1877), t. II, p. 36 (nous soulignons). Toutes les références qui renvoient à cette édition seront notées ainsi dans le texte: M. ii, 36 .

2 José-Michel Moureaux, L'CEdipe de Voltaire: introduction à une psycholecture (Paris: Minard, coll. 'Archives des lettres modernes 146', 1973), p. 6.

3 La Harpe, cité par Louis Dubois, dans les variantes de son édition d'CEdipe, dans CEuvres complètes de Voltaire (Paris: chez P. Mongie, t. III, 1828), p. 187.

4 René Pomeau, La Religion de Voltaire (Paris: Nizet, 1969), p. 85.

5 José-Michel Moureaux, op. cit., p. 55.

6 Ibid., p. 17.

7 lbid., p. 76.

8 lbid., p. 55.

9 La Harpe, cité par Louis Dubois, op. cit., p. 193.

10 Ibid.

11 René Pomeau, Voltaire en son temps, D'Arouet à Voltaire (1694-1734) (Oxford: Voltaire Foundation, 1985), p. 9.

12 René Pomeau, La Religion... op. cit., p. 76.

13 René Pomeau, D'A rouet à... op. cit., p. 105.

14 Ibid., pp. 280-81. 